\title{
Gene therapy for lung inflammatory diseases: not so far away?
}

\author{
Jean-Michel Sallenave, David J Porteous, Christopher Haslett
}

\begin{abstract}
The lung is a readily accessible target organ for gene therapy. To date, therapeutic gene delivery has largely focused on introducing functional, corrective genes in lung diseases arising from single gene defects such as cystic fibrosis. More recently interest has centred on gene therapy as a potential therapeutic tool in modulating complex pathological processes such as pulmonary inflammation. Genetic modification of critical components of the inflammatory process may be beneficial for example, overexpressing anti-elastase genes may circumvent elastase mediated lung damage in emphysema. With the development of improved viral and liposome vectors and the evolution of effective adjuvant immunosuppression to obviate host immune responses - for example, using selective cytokines and blockers of $T$ cell surface activation - the potential exists to target therapeutic doses of transgene to deficient or dysregulated cells. Furthermore, increased understanding of tissuespecific promoter regions and of mechanisms controlling regulation of gene expression offer the potential for close control of therapeutic gene expression within the lung. Continuing refinements in these technologies will provide new therapeutic strategies in inflammatory lung disease. (Thorax 1997;52:742-744)
\end{abstract}

Department of

Medicine,

University of

Edinburgh

Medical School,

Teviot Place,

Edinburgh EH8 9AG,

UK

J-M Sallenave

C Haslett

MRC Human Genetics

Unit,

Western General

Hospital,

Crewe Road,

Edinburgh EH2 2XU,

UK

D J Porteous

Correspondence to:

Dr J-M Sallenave.

Received 14 October 1996

Returned to authors

26 November 1996

Revised version received

30 April 1997

Accepted for publication

9 May 1997

The aim of somatic gene therapy is to replace, modify, or suppress the genes considered responsible for a given disease process. The lung is a particularly attractive organ for gene therapy because of its accessibility via the airways or the vasculature.

Cystic fibrosis and $\alpha_{1}$-antitrypsin $\left(\alpha_{1} \mathrm{AT}\right)$ deficiency are the two most common fatal single gene defects affecting the lung in the Western world. The genetic abnormalities in these conditions have been well characterised and led to these diseases becoming the first logical targets for lung-directed gene therapy. Indeed, several trials of specific gene replacement (using the adenovirus vector for gene carriage in the USA and liposome vectors in the UK) have shown promising results. ${ }^{1-3}$ The advances made in these areas have prompted interest in the role of gene therapy as a means of positively influencing more complex pulmonary disorders such as inflammatory lung diseases which might appear at first sight to be less amenable to genetic intervention. We shall confine our discussion to the potential application of gene therapy in inflammatory lung diseases (as an example of complex disease processes in the lung); however, it should be noted that farreaching and promising advances have been made in applying gene therapy to, for example, lung tumours and HIV infection.

\section{Scientific basis}

WHICH LUNG DISEASES MAY BE AMENABLE TO GENE THERAPY?

To date over 100 clinical trials of gene therapy for cancer have been conducted and reported, as well as 12 trials assessing gene therapy for HIV and a further 21 for single gene defects (mostly cystic fibrosis and $\alpha_{1}$ AT deficiency). However, as far as we are aware, no trials have assessed gene therapy for inflammatory lung diseases such as chronic obstructive pulmonary disease (COPD), adult respiratory distress syndrome (ARDS), and asthma.

The prevailing hypothesis is that inflammatory conditions such as COPD and ARDS arise from an imbalance between potentially injurious inflammatory agents (for example, neutrophil-derived elastase and reactive oxygen species) and tissue protective mechanisms. Much is now known about mechanisms of tissue defence and cytoprotection in these conditions, leading to our interest in the manipulation of these processes by gene therapy.
WHICH VECTORS ARE MOST APPROPRIATE IN TARGETING THE LUNG?

The main technical hurdle to be overcome is delivering the appropriate gene to the nucleus using a vector associated with minimal toxicity and maximal efficacy.

The armamentarium of vectors for gene delivery includes liposomes, viral vectors (retrovirus, adenovirus, vaccinia, herpes virus, and adeno-associated virus ${ }^{4}$, and molecular conjugates. ${ }^{4}$ In the context of the lung, liposomes and adenoviruses are particularly attractive. Cationic liposomes, while causing little or no 


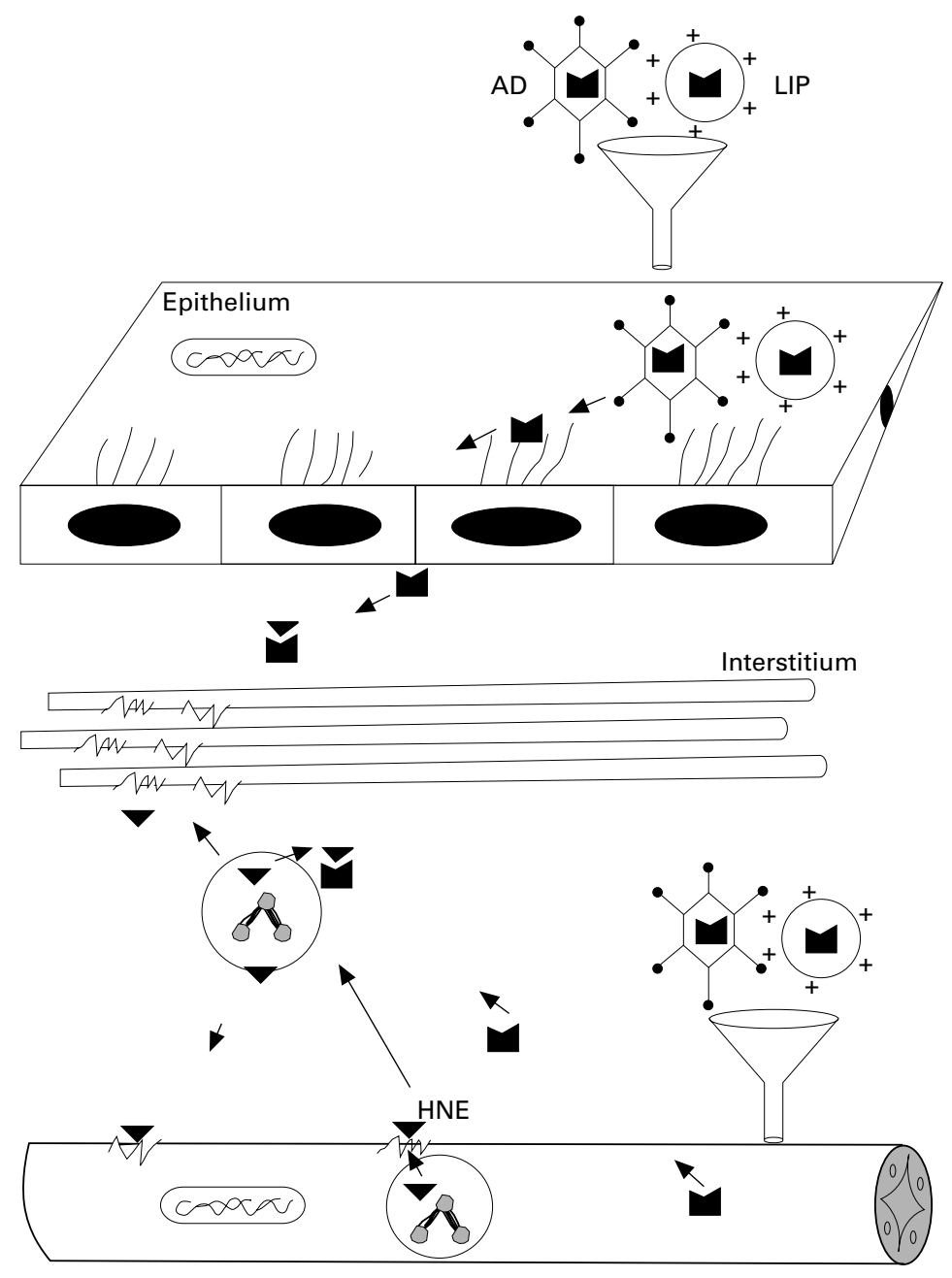

Endothelium

Figure 1 Gene therapy at a lung inflammatory locus. The adult respiratory distress syndrome (ARDS) is characterised by both a local and systemic inflammation and is triggered by a variety of stimuli, including bacterial endotoxin. Human neutrophil elastase (HNE, $\mathbf{\nabla}$ ) is one enzyme released by the activated neutrophil which is believed to be involved in the pathology by destroying both the interstitium and the endothelium. Protection against HNE could be provided by gene therapy using, for example, adenovirus (AD) and liposomes (LIP) to deliver genes for elastase inhibitors such as $\alpha_{1}$ proteinase inhibitor, secretory leucocyte proteinase inhibitor, or elafin $(\boldsymbol{\sim})$. Both the airway epithelium and the vasculature endothelium represent potential targets for gene therapy.

inflammation when administered in vivo, are in general poor gene transducers compared with adenoviruses. The latter have the opposite characteristics in that gene transduction is efficient (by virtue of mechanisms avoiding lysosomal degradation and promoting transfer to the nucleus) but significant inflammation and a specific immune response are induced in vivo.

Addition of specific peptides (growth factors, hormones, receptor ligands, or monoclonal antibodies) to liposome formulations can confer cell-specific targeting. ${ }^{5}$ Furthermore, the poor transducing capability of liposomes can be addressed by increasing nuclear tropism using nuclear localisation signals. Reduction of the pro-inflammatory and immunogenic properties of adenovirus vectors has been approached mainly by deleting some or all of the adenovirus genes ${ }^{67}$ and by manipulating the host immune response. The latter can be achieved by the use of conventional immunosuppressive drugs such as corticosteroids, cyclosporin, or FK506 or, more specifically, by using cytokines such as interleukin (IL) $-12^{8}$ which decreases neutralising IgA in the respiratory tract by switching off the CD $4+\mathrm{Th} 2$ response and by blocking co-stimulatory receptors on antigen presenting cells and lymphocytes such as B7/CD28 and CD40/CD40 ligand, thus reducing lymphocyte activation. Such approaches allow both sustained expression and safe repeated administration of the transgene. ${ }^{910}$

Further attempts to reduce host inflammatory responses and to enhance cell specificity and nuclear targeting are the focus of considerable research, and removal of the major hurdles toward effective, repeated gene therapy are keenly anticipated.

\section{Therapeutic potential}

The process of pulmonary inflammation is initiated by the stimulation of sequential migration of inflammatory cells from the circulation into the lung. Many pro-inflammatory molecules are potential targets for gene therapy. The influx of inflammatory cells could be prevented by counteracting the effects of selected cytokines and chemokines. For example, in situations where the "early" cytokines IL-1 and tumour necrosis factor (TNF) have been associated with adverse effects (such as in sepsis), overexpression of IL-1 receptor antagonist and soluble TNF receptor might be beneficial. Chemokines such as IL-8, responsible for the influx of neutrophils in ARDS, could be targeted using specific antisense DNA. Adhesion molecules present on inflammatory cells, endothelial cells, and epithelial cells could be downregulated.

Alternatively, overexpression of anti-inflammatory cytokines such as IL-10 could be beneficial in the resolution of inflammation. Indeed, recent in vivo work in rodents has shown that IL-10 can efficiently inhibit pulmonary inflammation by reducing both eosinophilia and neutrophilia, presumably, in the latter, through the promotion of apoptosis. ${ }^{11}$ Intervention by modulating intracellular factors can also be envisaged. For example, the nuclear factor NF- $\mathrm{NB}$, which is known to be upregulated during inflammation and involved in transcription of many inflammatory molecules (for example, IL-1, IL-6, IL-8, VCAM-1), has recently been targeted by the overexpression of its inhibitor $\mathrm{I} \kappa \mathrm{B} \alpha$. However, the relative lack of specificity of such nuclear factors must be carefully considered and may ultimately limit their clinical application.

For the approaches discussed to be successful in obviating lung inflammation, careful planning of the timing and duration of gene therapy will be vital. It will therefore be necessary to introduce mechanisms capable of "switching on" and "switching off" therapeutic genes. One such mechanism attracting attention involves the use of inducible promoters for expression of selected genes. Examples of such promoters include the heat shock, tetracycline- and 
steroid-responsive promoters. Furthermore, the use of lung-specific promoters such as surfactant protein $\mathrm{C}$ or Clara cell $10 \mathrm{kDa}$ promoters will ensure tissue-specific expression of therapeutic genes.

While there are clearly several attractions in aiming to attenuate the transmigration of inflammatory cells into tissues, a more effective and possibly safer strategy may be to inactivate the potentially deleterious enzymes and reactive oxygen species liberated by effector cells in situ. Animal models of pulmonary inflammation have clearly implicated the overexpression of elastase and reactive oxygen species in the development of lung injury suggesting that enhanced production of elastase inhibitors or oxidant scavenger molecules (such as superoxide dismutase) may have a significant protective effect. Indeed, several elastase inhibitors such as $\alpha_{1}$-antitrypsin, secretory leucocyte proteinase inhibitor (SLPI), and elafin have been extensively characterised ${ }^{12}$ and a gene augmentation approach might logically be expected to tip the inflammatory process in favour of protection rather than excessive tissue injury (fig 1).

\section{Conclusion}

Historically, emphasis in gene therapy has been directed towards treatment of single gene defects such as cystic fibrosis. We believe, however, that targeted gene therapy may, in the next few years, make a contribution to the treatment options for lung diseases with multifactorial aetiology such as COPD and ARDS. Key factors in the pathogenesis of these conditions have been identified (such as unregulated release of neutrophil elastase) and have suggested which specific genes may be of most therapeutic benefit. However, improvements require to be made in vector design, in ameliorating the specificity and durability of expression, and in manipulating the host immune response. At present liposomes and adenoviruses (or a combination of both) are the preferred vectors for use in the lungs, but if trials prove its safety then adeno-associated virus could offer an attractive alternative by virtue of its selective integration site in human chromosome 19, conferring stable and sustainable expression.

1 Crystal RG, McElvaney NG, Rosenfeld MA, Chu CS, Mastrangeli A, Hay JG, et al. Administration of an adenovirus containing the human CFTR CDNA to the respiratory tract of individuals with cystic fibrosis. Nature Genet 1994;8:42-51.

2 Caplen NJ, Alton EWFW, Middleton PG, Dorin JR, Stevenson BJ, Gao X, et al. Liposome-mediated CFTR gene transfer to the nasal epithelium of patients with cystic fibrosis. Nature Med 1995;1:3946.

3 Porteous DJ, Dorin JR, McLachlan G, Davidson-Smith H, Davidson $\mathrm{H}$, Stevenson BJ, et al. Evidence for safety and efficacy of DOTAP cationic liposome mediated CFTR efficacy of DOTAP cationic liposome mediated CFTR fibrosis. Gene Therapy 1997;4:210-8.

4 Curiel DT, Pilewski JM, Abelda, SM. Gene therapy approaches for inherited and acquired lung diseases. $A m \mathcal{F}$ proaches for inherited and acquired

5 Miller N, Vile R. Targeted vectors for gene therapy. FASEB f 1995;9:190-9.

6 Bramson JL, Graham FL, Gauldie J. The use of adenoviral vectors for gene therapy and gene transfer in vivo. Cur Opin Biotechnol 1995;6:590-5.

7 Fisher KJ, Choi H, Burda J, Chen SJ, Wilson J. Recombinant adenovirus deleted of all viral genes for gene therapy of cystic fibrosis. Virology 1996;217:11-22.

8 Yang Y, Trinchieri G, Wilson JM. Recombinant IL-12 prevents formation of blocking IgA antibodies to recombinan adenovirus and allows repeated gene therapy to mouse lung. Nature Med 1995;1:890-3.

9 Kay MA, Holterman AX, Mense L, Gown A, Ochs, HD Linsley PS, et al. Long term hepatic adenovirus-mediated gene expression in mice following CTLA4Ig administration. Nature Genet 1995;11:191-7.

10 Yang Y, Su Q, Grewal IS, Schilz R, Flavell RA, Wilson JM. Transient subversion of CD40 ligand function diminishes immune responses to adenovirus vectors in mouse liver immune responses to adenovirus vectors

11 Cox G. Interleukin-10 enhances resolution of pulmonary inflammation in vivo by promoting apoptosis of neutrophils. Am f Physiol (Lung Cell Mol Physiol) 1996;271 L566-71

12 Sallenave JM, Schulman J, Crossley J, Jordana M, Gauldie J. Regulation of secretory leukocyte proteinase inhibitor (SLPI) and elastase-specific inhibitor (ESI/Elafin) in human airway epithelial cells by cytokines and neutrophilic enzymes. Am f Respir Cell Mol Biol 1994;11:733-41. 